\title{
ABSTRACTS OF COMMUNICATIONS \\ Proceedings of the Twenty-Eighth Meeting of the Agricultural Research Modellers' Group
}

\author{
EDITED BY \\ J. FRANCE \\ Institute of Grassland and Environmental Research, North Wyke Research Station, Okehampton, Devon \\ EX20 $2 S B, U K$
}

This group, which is concerned with the applications of mathematics to agricultural science, is sponsored by the Biotechnology and Biological Sciences Research Council. It was formed in 1970, and has since met at approximately yearly intervals in London for one-day meetings. The twenty-eighth meeting of the group, chaired by Dr. D. A. Rose of the Department of Agricultural \& Environmental Science, University of Newcastle, was held in the Wellcome Meeting Room at the Royal Society, 6 Carlton House Terrace, London on Friday, 29 March 1996, when the following papers were read.

An investigation of the ventilation of a day-old chick transport vehicle. A. D. QUINN ${ }^{1}$ AND C. J. BAKER'. ${ }^{1}$ Silsoe Research Institute, Wrest Park, Silsoe, Bedfordshire MK45 $4 \mathrm{HS}, \mathrm{UK},{ }^{2}$ Department of Civil Engineering, The University of Nottingham, University Park, Nottingham NG7 2RD, UK

Computational fluid dynamics (CFD), a computer simulation system for predicting fluid flows, has successfully been applied to the ventilation problems encountered in offices and livestock buildings. These situations can generally be described as open space problems in which only certain specific zones of the domain are inhabited and air flow blockage is minimal. Studies into more complex situations, such as the ventilation of passenger vehicles, have also been undertaken with some success but have largely been unvalidated. This study seeks to investigate the potential of using a standard commercial CFD package in a closed and partially blocked situation in which uniformity of condition is required throughout the space. The validation of this model has been done using a statistical method based on full-scale experimental data. The case chosen was that of a dayold chick transport vehicle, a field in which a need has been identified for detailed information about ventilation effectiveness (Tamlyn \& Starr 1987).

The objectives of this project were to assess the ventilation achieved in the load of the vehicle and to use this information in the development of a CFD simulation. Experimental measurements of air velocity were collected in a full-scale model load space, with various configurations of load, using a three dimensional ultrasonic anemometer. CFD results of the same situations were obtained from a steady state, isothermal simulation. These sets of data were used to develop a statistical comparison method for quantitative validation of the CFD model.

The air velocity distribution within the load space was found to be a complex three dimensional composition of turbulent recirculating flow with jet inlets and associated regions of high shear. The presence of the load, in various configurations, had a channelling effect on the air flow through the load space. The predictions of mean velocity were found to be broadly in accordance with the experimental results, and statistical comparison showed a large majority to be within the experimental error of the observed values. Turbulence measures were not well predicted in most cases, particularly in the loaded situations. This was due to the lack of a turbulence model capable of incorporating the turbulence effects associated with the presence of the porous load.

TAMLYN, J. \& StaRR, J. R. (1987). Monitoring environment during transportation of day-olds. International Hatchery Practice 1(6), 11-17. 
Spatial dynamics in speciation. G. GARCÍARAMOS $^{1}$, F. SÁNCHEZ-GARDUÑO ${ }^{2}$ AND P. K. MAINI'. ' Department of Zoology, University of Texas, Austin, Texas 78712, USA, ${ }^{2}$ Departamento de Matemáticas, Facultad de Ciencias, UNAM Circuito Exterior, C.U., México 04510, D.F. Mexico, ${ }^{3}$ Centre for Mathematical Biology, Mathematical Institute, 24-29 St. Giles', Oxford OXI $3 L B, U K$

Species interaction resulting in a spatial distribution of different species has been widely investigated. Depending on this interaction, a new species introduced in a certain area may: (i) eliminate the ancestral species entirely, (ii) partially or totally overlap with it, or (iii) coexist with species segregation. Many theoretical models have been proposed for such behaviour but, whereas existing models can account for behaviours (i) and (ii), behaviour (iii) has eluded most models.

Models proposed for this type of interaction are typically of reaction-diffusion type, in which each species is assumed to disperse in response to gradients in density, and species interaction is modelled by competition. Extensions of such models, incorporating more complicated density-dependent dispersal, or allowing for environmental heterogeneity, have been analysed and shown not to exhibit species segregation.

A reaction-diffusion model for two species is presented (G. García-Ramos et al. 1996) in which the diffusion of each species depends on the gradient of the sum of the densities of both species as well as on the individual species density. Competition is modelled by Lotka-Volterra type dynamics, but environmental heterogeneity is included in the death term. The spatiotemporal dynamics of this coupled system of nonlinear partial differential equations is extensively analysed by numerical simulation. It is shown that this model can exhibit spatial segregation of species. It is also shown that if either of the conditions (density-dependent diffusion or environmental heterogeneity in the parameters) is relaxed, then the model is incapable of exhibiting species segregation and leads either to the extinction of one species or coexistence with spatial overlap. It is concluded that density-dependent diffusion and spatially varying parameters are essential to account for species segregation.

García-Ramos, G., SÁnchez-Garduño, F. \& Maini, P. K. (1996). Spatial segregation between sibling species, (preprint).
An empirical approach to predicting nitrate leached to surface water from catchments of mixed land use. S. L. CROPPER ${ }^{1}$, J. U. SMITH ${ }^{2}$, D. SCHOLEFIELD ${ }^{1}$, C. D. BROWN ${ }^{3}$ AND J. M. HOLLIS ${ }^{3} .{ }^{1}$ Institute of Grassland and Environmental Research, North Wyke Research Station, Okehampton, Devon EX20 2SB, UK, ${ }^{2} I A C R$ Rothamsted, Harpenden, Hertfordshire AL5 2JQ, $U K,{ }^{3}$ Soil Survey and Land Research Centre, Cranfield University, Silsoe, Bedfordshire MK45 $4 D T, U K$

Various models have been developed to predict nitrate leached from soil under particular land uses, usually at plot- and field-scales. However, these may be difficult to apply at other sites and over larger areas, because of the size and complexity of the data requirements and the involvement of different physical processes. These problems are compounded with the addition of often sophisticated hydrological models to simulate the transport of the leachate. This work is to address a requirement for a flexible system capable of modelling nitrate output from agricultural land to surface water, operating at differing scales up to the national level.

The approach uses simplified versions of field-scale nitrate models for pasture (NCYCLE, Scholefield et al. 1991) and for arable land (sUNDIAL, Smith et al. 1996), in combination with a UK-wide database of soil hydraulic characteristics. Spatial databases of soil and land use data, referenced to the National Grid, meet the reduced input requirements of the models. The baseflow index and standard percentage runoff coefficients are obtained similarly from the Hydrology of Soil Types (HOST) classification, derived at the catchment scale from soil and subsoil parameters and flow data (Boorman et al. 1995). The association is based on empirical relationships derived between leached load and peak concentration, which depend on certain soil characteristics, interpreted as influencing the degree of preferential, or bypass, flow (Scholefield 1996). This allows the determination of the load and peak concentration liable to be leached to surface water in any specified catchment.

The system has been developed initially to give calculations on an annual basis at a $1 \mathrm{~km}$ resolution. For practicality and flexibility, access to the models and data is being integrated within a geographical information system, allowing selection of catchments at different scales. The level of complexity in data input (of land use and soil type combinations) will be specified by the user and depend on the acceptable running time for the area selected and required accuracy of prediction. The models are now being modified to use daily or weekly precipitation data, to provide catchment-scale nitrate and flow data at shorter time scales. 
This work is funded by the Ministry of Agriculture, Fisheries and Food.

Boorman, D. B., Hollis, J. M. \& Lilly, A. (1995). Hydrology of soil types: a hydrologically-based classification of the soils of the United Kingdom. Report no. 126. Wallingford: Institute of Hydrology.

SCholefield, D., Lockyer, D. R., WhitehEad, D. C. \& TYSON, K. C. (1991). A model to predict transformations and losses of nitrogen in UK pastures grazed by beef cattle. Plant and Soil 132, 165-177.

SCholefield, D., LoRd, E. I., Rodda, H. J. E. \& WebB, B. W. (1996). Estimating peak nitrate concentrations from annual nitrate loads. Journal of Hydrology (in press).

Smith, J. U., Bradbury, N. J. \& AdDiscotT, T. M. (1996). SUNDIAL: SimUlation of Nitrogen Dynamics In Arable Land: a user-friendly, PC-based version of the Rothamsted nitrogen turnover model. Agronomy Journal (in press).

Neural networks as a modelling device. P. H. NYE. Department of Plant Sciences, University of Oxford, South Parks Road, Oxford OXI 3RB, UK

Neural networks consist of linked memory stores. They are so named by analogy with the brain which contains neurons linked by synapses. However, outside research on brain function, neural networks are increasingly used in recognition of patterns, for example, of signatures or speech, or in classification of organisms; in control systems, for example, of washing machines or agricultural implements; and in complex biological and environmental systems for predicting output from specified inputs - which is the topic of this paper. The way the variables in such systems interact is usually too uncertain to be expressed in mechanistic equations. Their effects are often non-linear and the result of feedback. Statistical and operational research methods such as linear programming or multiple polynomial regression cannot realistically be applied.

A back-propagation network is the commonest form (Wasserman 1989, 1993). It consists of a layer of input neurons, one for each input variable, and a layer of output neurons, one for each output variable. A 'hidden layer' of neurons lies between the input and output layers. Each of the input neurons is linked to all of the hidden neurons, and each of the hidden neurons is linked to all of the output neurons. The strength or 'weight' of the links is initially assigned randomly. The network is then 'trained' by giving it known inputs and comparing its outputs with those known by experiment or observation. The discrepancies are repeatedly used to adjust the weights till the discrepancies are reduced to an acceptable level hence 'back-propagation'. Once trained, a network can very rapidly predict output from any set of inputs lying within the training set. It is thus a very practical means of interpolation. But it is purely artificial, and gives no insight into mechanisms. Examples of applications in biology are presented.
Wasserman, P. D. (1989). Neural Computing. Theory and Practice. New York: Van Nostrand Rheinhold.

W asserman, P. D. (1993). Advanced Methods in Neural Computing. New York: Van Nostrand Rheinhold.

Markov Chain Monte Carlo methods (MCMC) for fitting spatio-temporal stochastic models of plant disease epidemics. G. J. GIBSON. Biomathematics \& Statistics Scotland, University of Edinburgh, James Clerk Maxwell Building, Mayfield Road, Edinburgh EH9 3JZ, UK

Understanding the spread of a plant disease requires consideration of both spatial and temporal dynamics. The interpretation of experimental observations of spread in space and time can be greatly assisted by the use of mathematical models. At the scale of the typical field experiment, where random events may dominate, the use of individual-based stochastic models is particularly appropriate. The paper describes how such models can be fitted to incidence data for plant diseases for which spatial or temporal sampling may be infrequent. The method, described in detail in Gibson (1996), extends previous work (Gibson \& Austin 1996) which tackled the problem using an alternative stochastic integration technique. Although the methods generalize, in this paper attention is restricted to simple epidemic models in which individuals are located at the vertices of a lattice, $L$. An infected individual at point $y$ infects a susceptible individual at $x$ at rate $F_{\alpha}(\boldsymbol{y}-\boldsymbol{x})$, where $\alpha$ is a vector of model parameters. This kind of model is meaningful in the study of, for example, the spread of a virus disease in a crop. Data describing the sets of diseased individuals $S_{0} \subset S_{1} \subset L$ observed at two times $t_{0}$ and $t_{1}$ respectively, where $t_{1}>t_{0}$, are considered. It is shown how, for a given model $F_{\alpha}$ and data of this form, MCMC methods can be used to make inference on the parameter vector $\alpha$. The method of the paper exploits the fact that for any temporal ordering, $\omega$, of the infections in $S_{1} \backslash S_{0}$ the posterior distribution $\pi(\alpha \mid \omega)$ can be computed exactly. When only initial and final states are observed, a Markov chain can be used to generate a sequence of samples $\left\{\left(\alpha_{i}, \omega_{i}\right)\right\}$ from the joint posterior distribution $\pi(\alpha, \omega)$ and the marginal distribution $\pi(\alpha)$ can be estimated by averaging $\pi\left(\alpha \mid \pi_{i}\right)$ over these samples. The technique is illustrated by using it to fit alternative stochastic models to observations of the spread of virus diseases in agricultural crops. Implications for disease control and experimental design are discussed.

Gibson, G. J. (1996). Markov chain Monte Carlo methods for fitting spatio-temporal stochastic models with applications in plant epidemiology. Applied Statistics (submitted).

Gibson, G. J. \& Austin, E. J. (1996). Fitting and testing spatio-temporal stochastic models with applications in plant epidemiology. Plant Pathology (in press). 
Modelling the dynamics of volatile fatty acids in the rumen of high-yielding dairy cows. A. BANNINK ${ }^{1}$, J. DIJKSTRA ${ }^{2}$ AND J. FRANCE ${ }^{2}{ }^{1}$ Institute for Animal Science and Health (ID-DLO), PO Box 65, 6500 AB Lelystad, The Netherlands, ${ }^{2}$ Institute for Grassland and Environmental Research, North Wyke Research Station, Okehampton, Devon EX20 2SB, $U K$

Volatile fatty acids (VFA) are the main energy fraction absorbed from the gastrointestinal tract of lactating dairy cows. Moreover, individual VFA have different fates in intermediary metabolism. Therefore, the precise molar proportion in which individual VFA are produced by rumen fermentation is an important determinant for the value of a feed in terms of nutrient availability for the animal. Several mechanistic rumen models have already been published that simulate rumen VFA production. These models represent rumen microbial metabolism in similar detail, but different concepts were adopted for this.

A comparison between the three most evolved models indicated large differences, although those rumen outputs that are generally measured in rumen digestion trials were predicted reasonably by all models. However, large differences in predicted amounts of fermented substrate, production rates of VFA and molar proportions of individual rumen VFA (Bannink \& De Visser 1996) are a portrayal of differences in the representation of the underlying mechanism. These results indicate that the models are calibrated to predict commonly observed rumen outputs reasonably, but differ on a more detailed level.

The model of Dijkstra et al. (1992) contains the most detailed representation of rumen fermentation processes. Although it predicted rumen degradation well under various conditions, predicted molar proportions of individual VFA remained inaccurate. In a subsequent simulation study, several components of the model, not directly involving microbial fermentation and growth, were modified to identify whether these components might be responsible for the large VFA prediction errors. It was established that modification of feed intake patterns, separation of insoluble, degradable substrate into a roughage and concentrate rumen pool, and inclusion of a mechanism for particle dynamics, had only a small effect on VFA predictions compared to the magnitude of their prediction errors. Improvement in their description can thus hardly contribute to a more accurate VFA prediction. On the other hand, prediction of VFA molar proportions was sensitive to the stoichiometry of VFA production from fermented substrate and VFA absorption from the rumen. Therefore, these two components need to be represented more precisely, but direct measurements of them in the rumen are absent and their apparent effect on measured rumen VFA contents is strongly interrelated. Such strong interrelationships between separate influencing factors are a major limitation on attempts to describe them independently from each other using literature data.

BANNINK, A. \& DE VISSER, H. (1996). Comparison of mechanistic rumen models on mathematical formulation of extra-microbial and microbial processes. Journal of Dairy Science (in press).

Dijkstra, J., Neal, H. D. St. C., Beever, D. E. \& France, J. (1992). Simulation of nutrient digestion, absorption and outflow in the rumen: model description. Journal of Nutrition 122, 2239-2256.

Desalinization of structured soils by intermittent leaching. M. AL-SIBAI, M. A. ADEY AND D. A. ROSE. Department of Agricultural \& Environmental Science, University of Newcastle, Newcastle upon Tyne NEI $7 R U, U K$

The only way to remove excess salts from soil effectively is by leaching, but ponding water on the soil surface and allowing infiltration consumes a large quantity of valuable water. During such leaching, water will flow preferentially through macropores between aggregates while the flow within aggregates will be much less. Consequently, solute within aggregates is removed much more slowly, thus decreasing overall leaching efficiency. For this reason intermittent ponding may be more efficient because it will allow time for solute to diffuse to the surfaces of aggregates during the rest period and subsequently be removed in macropore flow.

Our model simulates solute transport in structured soils under intermittent leaching. Solute movement during displacement is described by the mobileimmobile convection-dispersion equation (van Genuchten \& Wierenga 1976). During the rest period flow ceases, and solute redistributes within the aggregates by diffusion, the key variable being the effective diffusion coefficient, $D_{\mathrm{e}}$, of the solute in the aggregates. $D_{\mathrm{e}}$ was estimated for porous aggregates from observations of solute outflow into finite volumes of stirred distilled water. The model was verified by laboratory experiments with different aggregate-size distributions, flow velocities, and displacement and rest periods, with most of its parameters estimated independently. Water savings of $25 \%$ were possible, increasing as aggregate size, flow velocity and duration of rest period increased.

The model was modified to describe leaching when the macropores were drained at the end of each displacement. This was simulated by assuming that air displaced the soil solution by piston flow. The mass transfer coefficient, $\alpha$, between macro- and micropores was assumed to be zero when drained. However, when saturated, $\alpha$ was the key variable and was found by fitting the model predictions to our experimental results: $\alpha$ was not constant, but in- 
creased monotonically with the number of rest periods.

van Genuchten, M. T. \& Wierenga, P. J. (1976). Mass transfer studies in sorbing porous media: analytical solutions. Soil Science Society of America Journal 40, 473-480.

Modelling the effect of environmental conditions on the thermal balance of the ewe. J. R. TURNPENNY ${ }^{1}$, J. A. CLARK' ${ }^{\prime}$ AND A. J. MCARTHUR ${ }^{2}$ 'Department of Physiology \& Environmental Science, University of Nottingham, Sutton Bonington Campus, Loughborough LE12 5RD, UK, ${ }^{2}$ School of Pure \& Applied Sciences, The University of the South Pacific, Suva, Fiji

Knowledge of the thermal status of livestock is essential in determining an animal's productivity, growth rate, feed intake and ability to bear young, with all the attendant economic and welfare implications. A mathematical model has been developed to assess the thermal status of a ewe, by predicting metabolic rate and the occurrence of stress. The aims of the model are (i) to calculate heat loss from the model sheep using hourly meteorological data as input, (ii) to assess the degree of thermal stress, and (iii) to predict combinations of weather conditions which will produce a specified stress level.

The ewe was modelled as a system of cylinders with rounded ends (McArthur \& Monteith 1980). The model incorporates three layers - the underlying tissue, the fleece and the external environment. The transfer processes considered were conduction through the tissue, conduction, convection and thermal radiation through the coat, evaporation from the skin surface, and convection and thermal radiation from the coat surface. By specifying the thermal environment (in the form of hourly weather data) and feed intake, the computer calculated the temperatures of the layer interfaces by solving the energy balance at each interface. Hence the heat loss from the animal was calculated. Physiological responses to heat and cold stress, including panting, varying blood flow to the peripheries, shivering, and shade-seeking have been parameterized in the model.

The model provides a good simulation of sample data from real sheep both in the calorimeter and in the field, in all components of the energy balance. Results of the simulations are presented. Solar radiation was found to be an important term in the energy balance at the coat surface. Further work and use of the model is discussed, especially its part in a major project to assess the impact of climate change on animal production in UK agriculture.

We thank the Ministry of Agriculture, Fisheries and Food for funding this work.

MCARThur, A. J. \& Monterth, J. L. (1980). Air movement and heat loss from sheep. 1. Boundary layer insulation of a model sheep, with and without fleece. Proceedings of the Royal Society of London, Series B 209, 187-208.

Predicting the vegetation on ex-arable land in Britain. N. E. ELLIS, L. G. FIRBANK, M. O. HILL AND R. D. SWETNAM. Institute of Terrestrial Ecology, Monks Wood, Abbots Ripton, Huntingdon, Cambs PE17 2LS, UK

A model has been built which predicts and forecasts the plant species most likely to occur within naturally regenerating vegetation on ex-arable sites within Britain. Such a model is valuable in considering different management options for set-aside land. There were five parts to the modelling.

(1) The distribution of species throughout Britain were mapped using data collected in a systematic survey by the Botanical Society of the British Isles (BSBI) (Palmer \& Bratton 1995). Probabilities of finding a given species within each $2 \mathrm{~km}$ square (tetrads) in Britain were extrapolated from the 788 presence/absence records of the BSBI survey. The probability of finding a given species in each tetrad was estimated as a weighted mean local frequency. Weights were defined by the bivariate Gaussian function with a root-mean-square deviation of $30 \mathrm{~km}$ (Le Duc et al. 1992a). Since the inclusion of soil data significantly improves predictions (Le Duc et al. $1992 b$ ), frequencies of occurrence of a species on soil subgroups were calculated using the Soil Survey and Land Research Centre dataset (SSEW 1983; SSS 1984). A logistic regression produced parameters for each species of $(a)$ detecting the species in a tetrad on the basis of location and $(b)$ detecting the species in a tetrad according to the species affinity to the soil subgroups in that square (Le Duc et al. 1992b). These parameters provide the probability of detecting a given species in any given tetrad in Britain $\left(P_{\text {anyhabitat }}\right)$.

(2) The $P_{\text {anyhabitat }}$ occurrences were used to provide a list of species for any given site.

(3) The probability of occurrence in first year setaside $\left(p^{*}\right)$ was calculated for 110 species using data from Critchley \& Smart (1995). The probability of occurrence in first year set-aside per tetrad $\left(P_{\text {ex-arable }}\right)$ was calculated by multiplying $P_{\text {anyhabitat }}$ by $p^{*}$.

(4) A Fortran model (Hill 1992) takes the species list $P_{\text {ex-arable }}$ and uses 26 life history attributes of each species to predict vegetation succession. Attributes include germination characteristics, growth rate, litter and seed production. The change in vegetation groups (e.g. perennial broadleaves, annual grasses, etc) for a given time (up to 10 years) are produced. Simple management options are available, e.g. cultivation in March, cut in May/July, leave cuttings.

(5) The model is being validated against vegetation and management data collected from set-aside land.

Funding from the Ministry of Agriculture, Food and Fisheries is gratefully acknowledged. 
Critchley, C. N. R. \& Smart, S. (1995). Botanical Evaluation of Set-aside Land in England II. Plant Communities and Successional Change. Newcastle: ADAS.

HILL, M. O. (1992). Modelling vegetation succession in abandoned arable fields in Britain. Coenoses 7, 153-159.

Palmer, M. O. \& Bratton J. H. (1995). A sample flora of Britain and Ireland. The Botanical Society of the British Isles Monitoring Scheme. Peterborough: Joint Nature Conservation Committee.

Le Duc, M. G., Hill, M. O. \& Sparks, T. H. (1992a). A method for predicting the probability of species occurrence using data from systematic surveys. Watsonia 19, 97-105.

Le Duc, M. G., Sparks, T. H. \& Hill, M. O. (1992b). Predicting potential plant colonisers of new woodland plantations. Aspects of Applied Biology 29, 41-48.

SOIL SUR VEY OF ENGLAND AND WALES (1983). $1: 250000$ Soil Map of England and Wales (6 Sheets); and Legend for the I:250000 Soil Maps (A Brief Explanation of the Constituent Soil Allocations). Harpenden: Rothamsted Experimental Station.

SoIL SURVEY OF SCOTLAND (1984). Organisation and Methods of the 1:25000 Soil Survey of Scotland. Aberdeen: The Macaulay Institute for Soil Research.

Mathematical and simulation modelling of root system architecture. A. J. F. BUCKNER, J. E. HOOKER AND K. E. BLACK. Soil Biology Unit, Land Resources Department, Scottish Agricultural College, Craibstone Estate, Aberdeen AB2 9TQ, UK

The branched, hierarchical nature of plant root systems is a factor that greatly complicates attempts to model them mathematically, since individual roots are not necessarily independent of one another: death of a root automatically entails the loss of all roots that have branched from it. Many researchers have neglected root architecture (the division of root systems into primary roots, first order laterals, second order laterals, etc) in their models. However, many factors, for example mycorrhizal colonization (Hooker et al. 1992), are known to affect root system architecture, which will then in turn affect the growth, form and functioning of the root system. An appropriate modelling strategy would clearly be of use in determining the effect of factors affecting root processes on root system dynamics. Several detailed root system computer simulation models, for example ROOTMAP (Diggle 1988), have been published but none of these allows for root mortality. Their applicability and usefulness are thus limited since root longevities may be very short (Hooker et al. 1995).

The present study has involved the construction of two temporal models of root system dynamics, both of which account for root architecture. The first is a deterministic mathematical model, the second a Monte Carlo computer simulation model. Both models incorporate root extension, branching, mortality and the loss of roots of one order due to the death of roots of a lower order (root loss). The parameters controlling the rates of these processes may vary with age and time, so allowing environmental modifying factors to be incorporated. The original purpose of the simulation code was to investigate the validity of assumptions made by the deterministic model, such as whether the discrete process of root branching can be represented as a continuous process and whether root loss is taken into account correctly. However, the simulator should prove highly useful in its own right, due to its higher level of detail and the ease with which spatial effects may be incorporated. Possible applications of the models include studies of root turnover, the dynamics of fungal infection and the influence of mycorrhizal colonisation on the evolution of root system form.

Diggle, A. J. (1988). ROOTMAP - a model in threedimensional coordinates of the growth and structure of fibrous root systems. Plant and Soil 105, 169-178.

HoOKER, J. E., MUNRo M. \& AtKinson, D. (1992). Vesiculararbuscular mycorrhizal fungi induced alteration to poplar root system morphology. Plant and Soil 145, 207-214.

Hooker, J. E., Black, K. E., Perry, R. L. \& Atrinson, D. (1995). Arbuscular mycorrhizal fungi induced alteration to root longevity of poplar. Plant and Soil 172, 327-329. 